\title{
Bilateral tears of the retinal pigment epithelium
}

\author{
KAZUMI NOROSE,' TSUYOSHI TANINO, ' AND KATSUZO SEGAWA' \\ From the 'Department of Ophthalmology, Shinshu University School of Medicine, Matsumoto, and the \\ 'Department of Ophthalmology, Kanto Teishin Hospital, Tokyo, Japan
}

SUMmaRY This paper describes bilateral tears of the retinal pigment epithelium (RPE) which followed photocoagulation treatment of retinal pigment epithelial detachment (PED) in the right eye and which occurred spontaneously in the left eye. A 66-year-old Japanese male presented with PED approximately $4 \times 6$ disc diameters in size in his right eye, and one disc diameter in the left eye, both in the macular regions. The right PED was treated by placing a row of argon laser photocoagulation along the margin of the PED, with avoidance of the papillomacular bundle. Five days after treatment the PED enlarged peripherally beyond the coagulated site. Nineteen days later an RPE tear developed at the temporal edge of the detachment in the right eye. The remaining detached RPE retracted centrally, forming curled folds. About 40 days after the initial examination the patient complained of sudden reduction of vision in his left eye. At this time an enlargement of the PED was found in his left eye. One week after this examination a small RPE tear was found in the nasal border of the PED in the left eye. The tear in the left eye gradually developed centrally to the fovea.

Senile macular degeneration has recently become a major cause of visual loss in the elderly population.' Detachment of retinal pigment epithelium (PED) associated with senile macular degeneration is frequently reported. ${ }^{2}$ Hoskin et al. ${ }^{3}$ described RPE tears as a newly recognised severe complication of PEDs in senile macular degeneration. The RPE tear is reported as occurring spontaneously or after laser photocoagulation. ${ }^{3-17}$

There are many reports ${ }^{3811}$ on the relationship between laser photocoagulation to the PED and the pathogenesis of the RPE tears. However, the mechanism of the PRE tear has not been clarified.

In this report we describe bilateral tears of the RPE which occurred after argon laser photocoagulation to the PED in the right eye and spontaneously without any treatment in the left eye with PED.

\section{Case report}

A 66-year-old man visited our clinic on 13 December 1985 complaining of metamorphopsia in the right eye of two weeks' duration. He was in good health and had no previous eye problems. His visual acuity was $0.1(\mathrm{nc})$ in the right eye and $0.7(1.0 \times+0.5 \mathrm{D})$ in the

Correspondence to Kazumi Norose, MD. left eye. The external eye examination, pupillary responses, intraocular pressure, and results of slitlamp examinations were normal in both eyes except for mild cataract. Pertinent findings were confined to the funduscopic examination. He had a large serous detachment of the pigment epithelium, oval in shape, which measured $4 \times 6$ disc diameters (DD) in the right macula, and a small round serous detachment of the pigment epithelium measuring $1 \mathrm{DD}$ associated with drusen in the left macula. The discs and vessels were normal in both eyes. Fluorescein angiography confirmed the presence of PED and showed a delay in the overall fluorescein staining (Figs. 1A, B).

On 20 December 1985 argon laser photocoagulation was placed along the margins of the PED, with sparing of the papillomacular bundle in the right eye (44 shots with a spot size of $200 \mu \mathrm{m}$, a duration of $0 \cdot 2 \mathrm{~s}$, and a setting of $200 \mathrm{~mW}$ ).

Five days after photocoagulation the PED enlarged in the right eye over the photocoagulation scar. Fluorescein angiography showed extensive hyperfluorescent areas along the margins of the PED. Corticosteroids were administered orally for 120 days. Nineteen days after the laser treatment a large RPE tear was demonstrated in the temporal margin of the PED, crescentic in shape, and measuring $4 \times 0.5 \mathrm{DD}$ (Fig. 2). The remaining detached RPE 


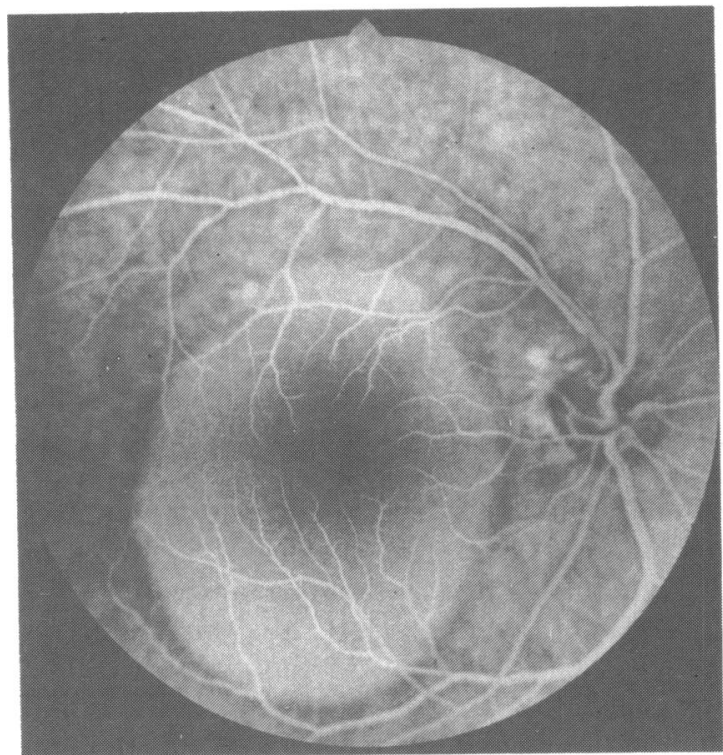

Fig. 1A

Fig. 1 A: Fluorescein angiogram in the right eye shows large retinal pigment epithelial detachment (PED) in the macular region. B: Fluorescein angiogram in the left eye shows small PED associated with drusen. 13 Dec 85.

retracted, centrally forming curled folds. Large choroidal blood vessels could be seen clearly through the tear. The free edge of the tear was not visible in the subretinal space because it retracted and infolded under the remaining elevated pigment epithelum. There was a serous detachment of the retina overlying the pigment epithelial defect.

Fluorescein angiography of 8 January 1986 gave characteristic findings. In the arterial phase hyperfluorescence of the well defined area was observed through the exposed Bruch's membrane and choroid. Within several seconds this area became intensely hyperfluorescent. The retracted area of pigment epithelium remained hypofluorescent until the late phases of the angiogram. Approximately 1 minute after the injection, dye leaked from the RPE tear into the subretinal space. There was no faint mottled fluorescein staining on the surface of the pigmented mound.

The serous retinal detachment gradually resolved.

On 22 January 1986, six weeks after his initial visit, the patient noted sudden deterioration of vision in the left eye. At that time his visual acuity was 0.04 $(0 \cdot 1 \times 1 \cdot 5 \mathrm{D})$ OS. Fundus examination showed a large round $\mathrm{PED}$, measured $6 \times 8 \mathrm{DD}$ in the left macula.

In the PED lesion there was a small excavated zone corresponding to bare Bruch's membrane at the nasal

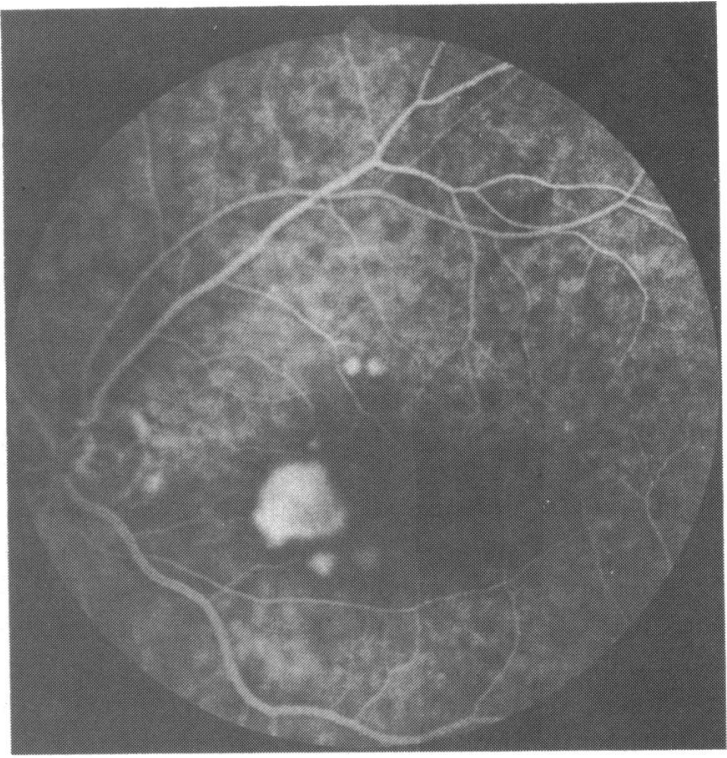

Fig. 1B

edge of the PED. Fluorescein angiography showed a well circumscribed zone of uniform hyperfluorescein corresponding to the defect of the RPE (Fig. 3). The RPE was torn along the infranasal margin of the detachment, with retraction noted supratemporally. During the next four months the tears gradually extended to the centre of the fovea (Fig. 4). Steroid therapy was reduced gradually because the effect was not clear. On 12 September 1986, approximately nine months after the initial onset of symptom, the patient's visual acuity in the right eye had improved to $0.2(0.6 \times+1.5 \mathrm{D})$, and that in the left eye to 0.3 $(0.8 \times+1.5 \mathrm{D})$. On funduscopic examination the shape and the size of the RPE tears were unchanged except for a subretinal translucent layer of tissue within the RPE tears (Figs. 5A, B). There was no evidence of subretinal neovascularisation biomicroscopically.

Goldmann perimetry revealed normal peripheral fields with paracentral absolute scotoma which corresponded to the RPE tears in both eyes. The areas of absolute scotoma gradually became smaller and improved to become relative scotoma.

The electroretinogram (ERG) showed normal amplitudes of $a$ and $b$ waves in both eyes. The electro-oculogram (EOG) showed a light-peak/darkthrough ratio of 1.70 on 6 February 1986 in both eyes. On 9 June 1986 it showed 1.80 in the right eye and 1.70 in the left (normal ratio $>1.85$ ).

Blood and urine examination showed no abnormality. 


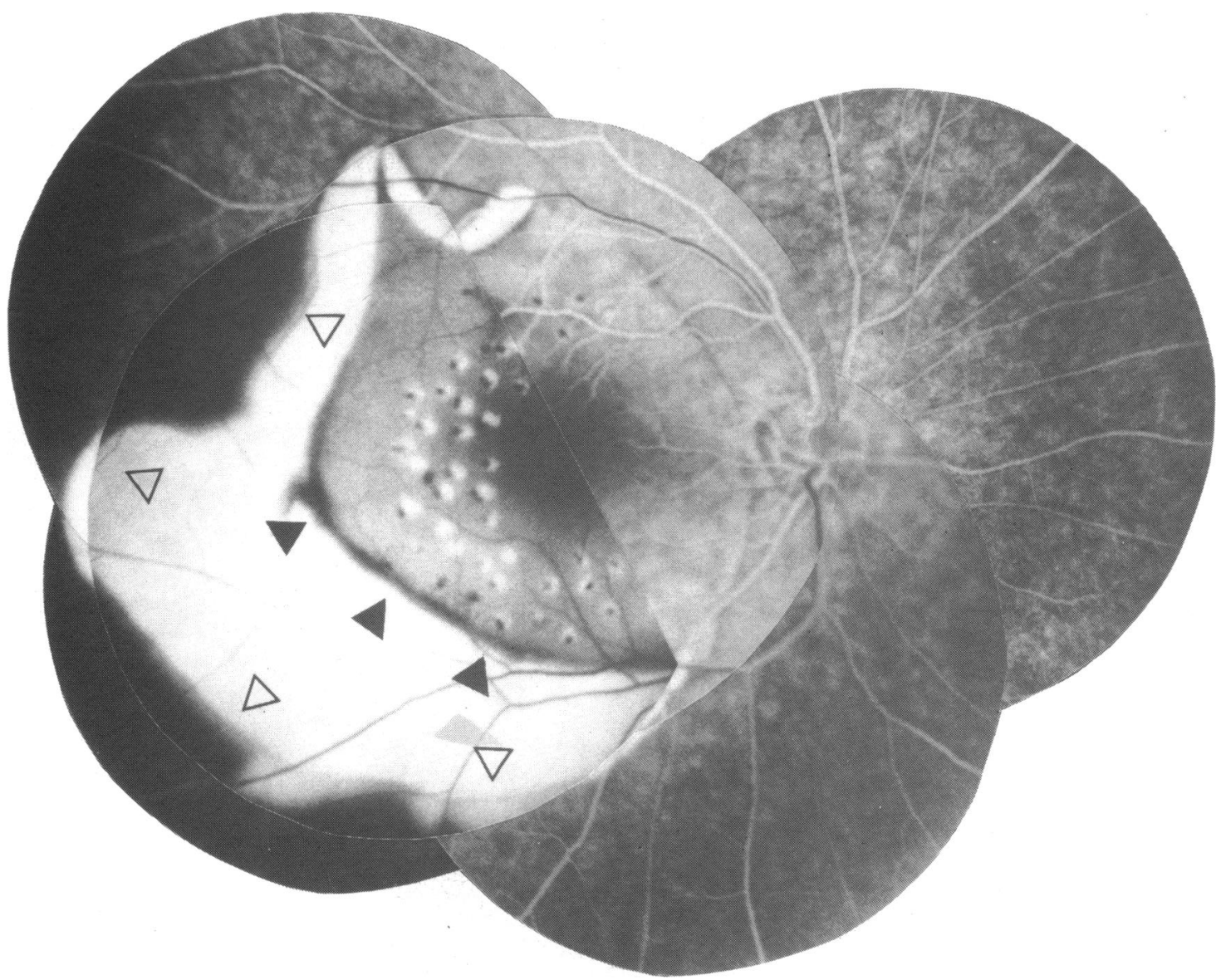

Fig. 2 Post-treatment angiogram in the right eye shows the enlarged retinal pigment epithelial detachment (PED) over the photocoagulation scar and large tear in the retinal pigment epithelium (RPE) along inferior border of detachment (white arrows). Free edge of torn RPE is folded beneath remaining PED (black arrows), and retracted pigment epithelium remains hypofluorescent. 13 Jan 86.

\section{Discussion}

Serous PED is a common finding in patients with senile macular degeneration. ${ }^{1}$ Approximately $30 \%$ of the patients who have PEDs have bilateral ones. ${ }^{18}$

Although in the past senile macular degeneration was not so commonly found in Japan as in the USA or Europe, it has recently become one of the main diseases causing blindness. Further, there are many reports of bilateral senile macular degeneration in Japan. ${ }^{19} 20$

RPE tears have recently been described as a new, uncommon, but serious complication of the PED..$^{3-17}$ They occur in approximately $10 \%$ of PEDs, ${ }^{5}$ and bilateral RPE tears have been shown to have almost similar findings and courses. ${ }^{34610}$ Previously they were often mistakenly diagnosed as pigment epithelial atrophy or choroidal neovascularisation.
The course of the PEDs and tears in our case was observed in detail in both eyes. Particularly in the left eye, the whole natural course of the RPE tear formation was revealed, developing first from the small PED to the large one and finally to the RPE tear. RPE tears have a characteristic clinical and angiographic appearance. ${ }^{310}$ They show a flat, uniform area of exposed choroid and an area of pigment epithelial elevation. The area of denuded choroid and Bruch's membrane appears hyperfluorescent during fluorescein angiography. By contrast, the area of infolded pigment epithelium shows hypofluorescence.

Our case had characteristic fundus features. The PED in the right eye enlarged after argon laser photocoagulation, and the RPE tear occurred along the temporal margin of the enlarged PED. On the other hand a spontaneous tear was seen in the left 
Fig. 3 Fluorescein angiogram in the left eye shows sharply outlined margin of retinal pigment epithelial tear. 22 Jan 86.

Fig. 4 Fluorescein angiogram in the left eye shows hyperfluorescence of exposed Bruch's membrane, choroid, and folded edge of torn RPE. $20 \mathrm{Feb} 86$.

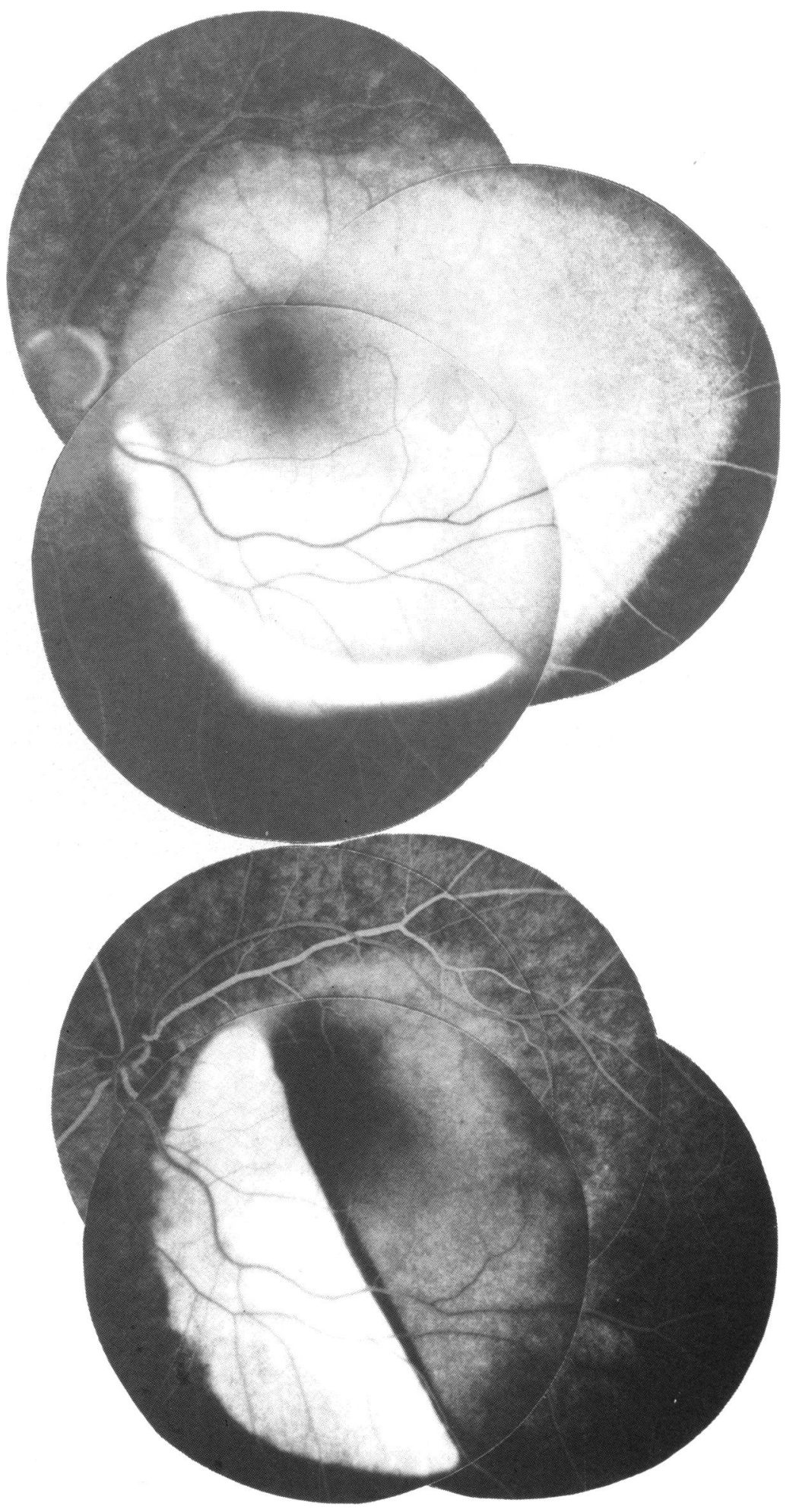




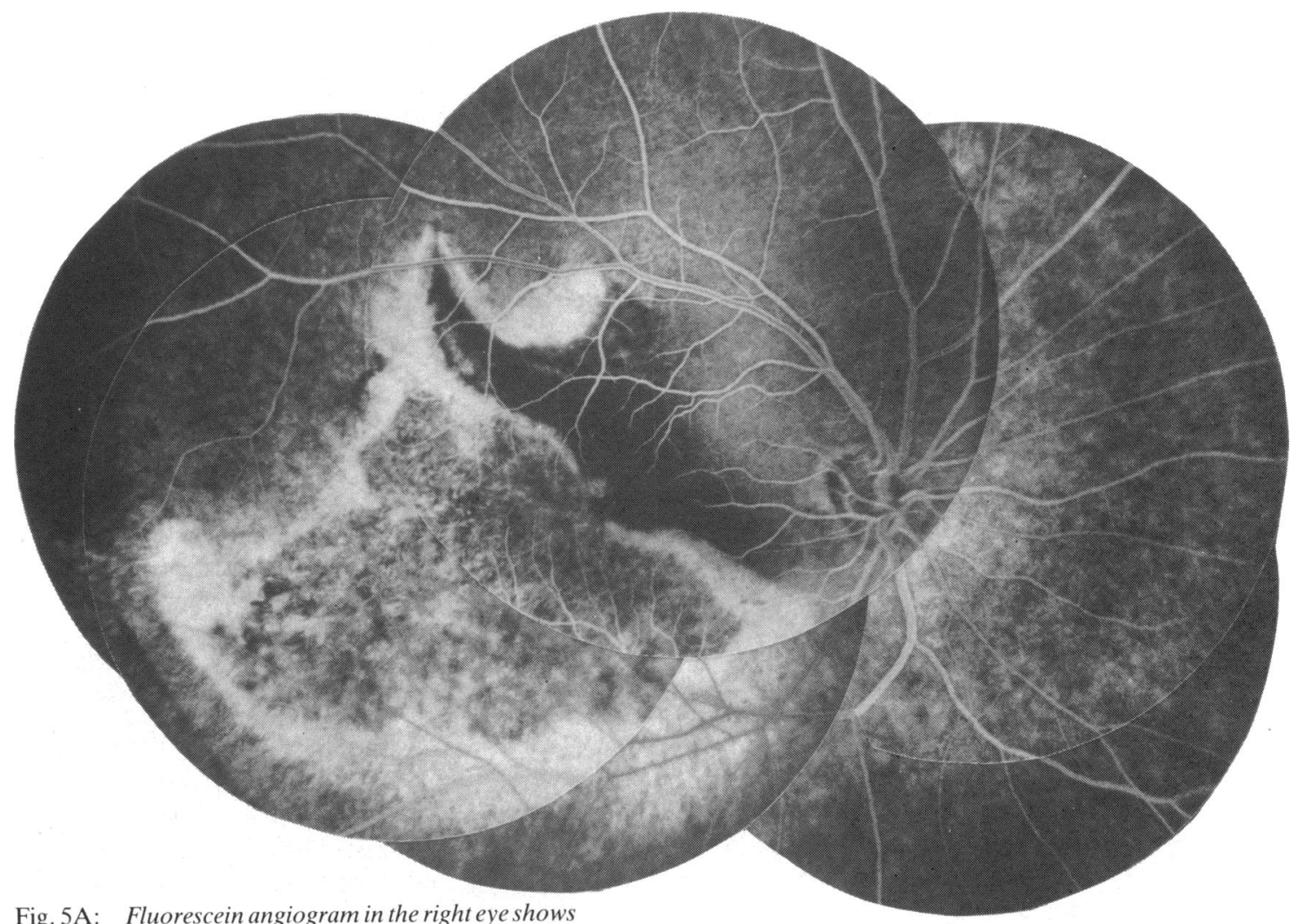

igment epithelial elevation along temporal margin. 12 Sept 86.

eye. But the course and fundus appearance of the RPE tears were essentially similar in both eyes during the follow-up period.

Although photocoagulation has been used to flatten the PED," there are many reports ${ }^{\times 112}$ of tearing following laser photocoagulation over the surface of the PEDs. A recent controlled study of elderly patients treated with argon laser photocoagulation" implied that this form of therapy confers no visual benefit and that tearing of the pigment epithelium occurs earlier in the treated eyes than in the untreated. RPE tears are the result of tractional forces acting along the margin of the PED, and the risk of tearing is greatly enhanced by laser photocoagulation..$^{48}$

Our case demonstrated essentially the same evolution of the RPE tears in both eyes, though the PED of the right eye was treated with laser photocoagulation and the left was not. Argon laser photocoagulation may further weaken the RPE along the margin of the PED. ${ }^{21}$ But in our case in spite of the laser treatment the tear in the right eye still occurred in the margins of the enlarged PED, not along the area of argon laser photocoagulation. So far as our observations go, the photocoagulation to the right eye may not be the essential factor producing the RPE tear.

Hyperfluorescent areas of leakage into the subretinal space along some margins of the PED represent an area of damaged or degenerating pigment epithelium without neovascularisation that may be more likely to tear." ${ }^{17}$ These phenomena may demonstrate the importance of bending forces at the detachment margin. Krishan et al." proposed that mechanical forces make the detachment margin the site of greatest bending stress and the most likely site for the tear. ${ }^{+}$The findings in our case appear to support the idea that mechanical factors have a more important role in the pathogenesis of RPE tears." The tears occurred as soon as the PEDs were enlarged in both eyes. So the larger size of PEDs may also be important in the pathogenesis of the tear. ${ }^{+}$

The precise reasons for RPE tearing are as yet unknown. Hoskin et al. ${ }^{3}$ argued from angiographic observations that separate cleavage planes may coexist, one between pigment epithelium and its basement membrane, the other between basement 


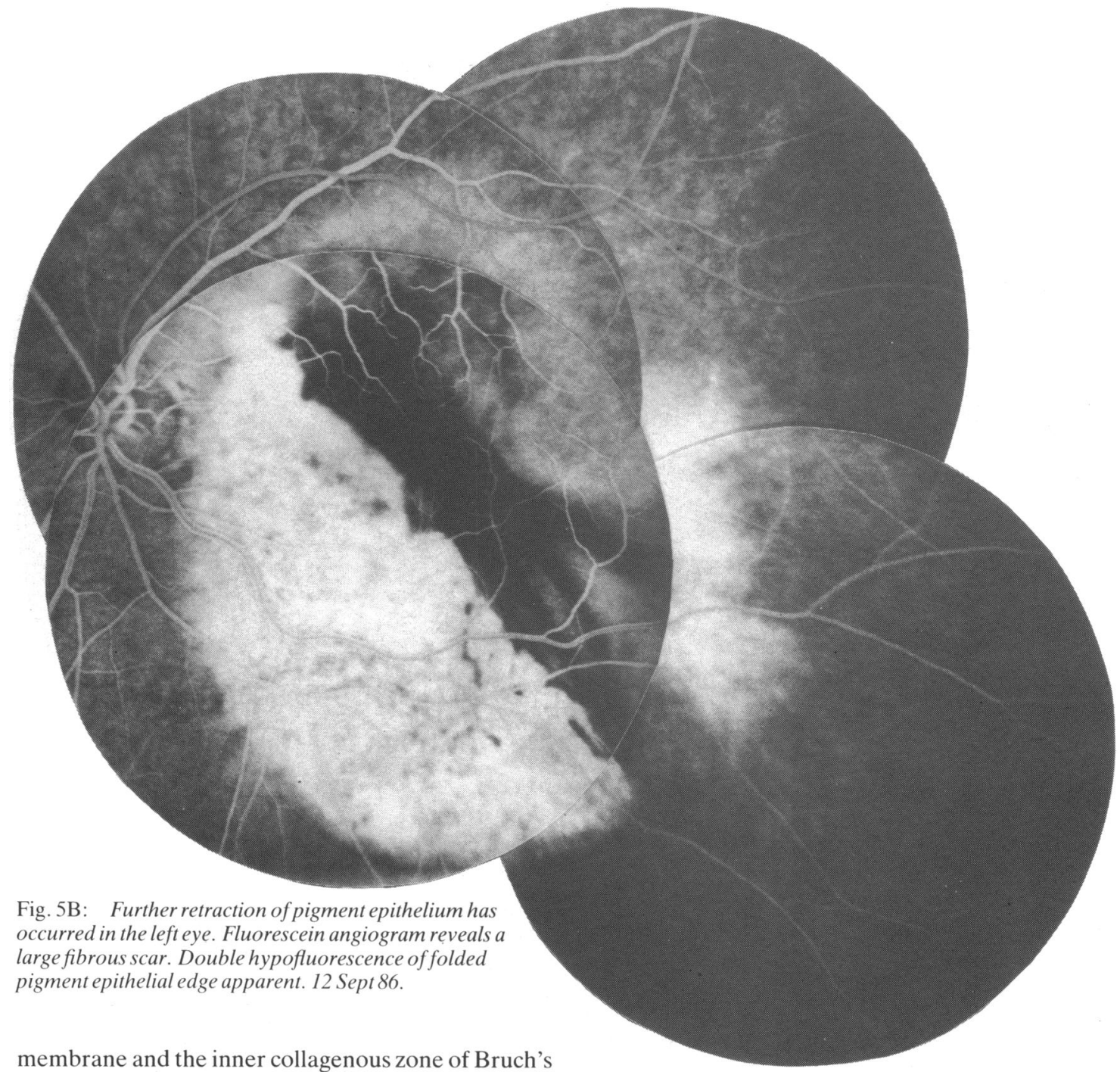

membrane. They have suggested that RPE tears occur in the RPE that is separated from its basement membrane. They noted that the RPE in the area of the tear has a smooth, featureless appearance, and on fluorescein angiography showed uniform fluorescence, suggesting the absence of material on the under surface of the PED. Green and Yarian ${ }^{9}$ believed that the posterior curling of the torn edge of the RPE contradicts Hoskin and colleagues' hypothesis and is further evidence that the basement membrane is adherent to the elevated torn edge of the RPE. Adjacent retinal pigment epithelial cells are joined firmly together by zonula occludentes that encircle each retinal pigment epithelial cell at its apex. ${ }^{49}$ Gass $^{7}$ confirmed that the separation of the pigment epithelium from its basement membrane is not responsible for the uniform fluorescence and that its predisposing the pigment epithelium to tear is not supported by evidence from the light microscope, electron microscope, or clinical examination. Clinicopathological correlations are needed to settle this question.

The outer blood-retina barrier is disrupted because of the creation of a large defect in the pigment epithelium. ${ }^{+}$The dehydration of the subretinal space can no longer act in the area of the tear, and a serous retinal detachment would result. In most cases with the RPE tears, however, fluorescein leakage into the subretinal space from the area of exposed Bruch's membrane and choroid is minimal. In our case the subretinal exudate also disappeared in a few weeks. Other forces, perhaps hydrostatic in nature, may be 
more important in maintaining retinal aposition. ${ }^{4}$ With time, the RPE may proliferate, ${ }^{7}$ closing the defect and re-establishing the blood-retina barrier. ${ }^{4}$ Once re-epithelialisation occurs, the subretinal exudate disappears.' Studies ${ }^{13}$ have shown that atrophic changes occur in the choriocapillaris during the natural course of macular degeneration. These atrophic changes may be a factor in the less than expected amount of leakage that is observed in these cases. $^{6}$

So far as we know, there are no reports on visual field changes in association with the RPE tears. In our case the absolute scotoma was observed corresponding to the RPE tear in the early stage, and it was enlarged with the development of the tear. But the area of absolute scotoma became smaller, and then relative when the tear was covered with the fibrous tissue. This may suggest that the function of photoreceptors is soon recovered after RPE tears.

In the present study the ERG excluded widespread impairment of sensory retina. The EOG showed an abnormal EOG ratio, and further EOG study of the PED or the RPE tear is necessary to evaluate the RPE function.

Decker et al. $^{6}$ reported that $30 \%$ of PEDs developed subretinal new vessels. Subpigment epithelial choroidal neovascularisation has been suggested the primary cause of the detachment and tear in the pigment epithelium. ${ }^{7}$ The present case showed neither choroidal neovascularisation nor subretinal or subpigmentary epithelial haemorrhage.

There is no treatment of confirmed value for RPE tears, but there is a report ${ }^{14}$ stating that photocoagulation is recommended for their early treatment when they are associated with serous sensory retinal detachment.

\section{References}

1 Gass JDM, Norton EWD, Justice J. Serous detachment of the retinal pigment epithelium, Ophthalmology 1966; 70: 990-1015.

2 Meredith TA, Braley RE, Aaberg TM. Natural history of serous detachments of the retinal pigment epithelium. Am J Ophthalmol 1979; 88: 643-51.
3 Hoskin A, Bird AC, Sehmi K. Tears of detached retinal pigment epithelium. Br J Ophthalmol 1981; 65: 417-22.

4 Cantrill HL, Ramsay RC, Knobloch WH. Rips in the pigment epithelium. Arch Ophthalmol 1983; 101: 1074-9.

5 Casswell AG, Kohen D, Bird AC. Retinal pigment epithelial detachments in the elderly: classification and outcome. $\mathrm{Br} J$ Ophthalmol 1985; 69: 397-403.

6 Decker WL, Sanborn GE, Ridley M, Annesley WH, Sorr EM. Retinal pigment epithelial tears. Ophthalmology 1983; 90: 507-12.

7 Gass JDM. Pathogenesis of tears of the retinal pigment epithelium. Br J Ophthalmol 1984; 68: 513-9.

8 Gass JDM. Retinal pigment epithelial rip during krypton red laser photocoagulation. Am J Ophthalmol 1984; 98: 700-6.

9 Green SN, Yarian D. Acute tear of the retinal pigment epithelium. Retina 1983; 3: 16-20.

10 Krishan NR, Chandra SR, Stevens TS. Diagnosis and pathogenesis of retinal pigment epithelial tears. Am J Ophthalmol 1985; 100: 698-707.

11 Moorfields macular study group. Retinal pigment epithelial detachments in the elderly: a controlled trial of argon laser photocoagulation. BrJ Ophthalmol 1982; 66: 1-16.

12 Okada $\mathrm{H}$, Ohkuma $\mathrm{H}$, Uyama $\mathrm{M}$. Tear of the retinal pigment epithelium following argon laser photocoagulation. Jpn J Clin Ophthalmol 1985; 39: 1145-9.

13 Sarks SH. Ageing and degeneration in the macular region: a clinicopathological study. Br $j$ Ophthalmol 1976; 60: 324-41.

14 Sunakawa M, Tsukahara I. Tear of the retinal pigment epithelium and serous retinal detachment. Am J Ophthalmol 1985; 100: 488-9.

15 Swanson DE, Kalina RE, Guzak SV. Tears of the retinal pigment epithelium. Retina 1984; 4: 115-8.

16 Yamamoto K, Kanai K. Okada H, Uyama M. Spontancous tear of retinal pigment epithelium. Report of a case. Jpn J Clin Ophthalmol 1985; 39: 1092-3.

17 Bird AC, Sehmi KS. Tears of detached retinal pigment epithelium. Trans New Orleans Acad Ophthalmol 1983;317-25. Symposium on medical and surgical diseases of the retina and vitreous. St Louis: Mosby, 1983.

18 Elman MJ, Fine SL, Murphy RP, Patz A, Auer C. The natural history of serous retinal pigment epithelium detachment in patients with age-related macular degeneration. Ophthalmology 1986; 93: 224-30.

19 Miyake K, Miyake C, Sakamura S, Miura H. Natural history of senile disciform macular degeneration in Japanese. Folia Ophthalmol Jpn 1977; 28: 308-20.

20 Mine N. Clinical study of senile disciform macular degeneration. Nippon Ganka Gakkai Zasshi 1986; 90: 398-408.

21 Wallow IHL, Tso MOM, Elgin S. Experimental photocoagulation of the human retina: II. Electron microscopic study. Arch Ophthalmol 1977; 95: 1041-50.

Accepted for publication 4 June 1987. 